\title{
Mixed Self-Assembled Monolayers of Semirigid Tetrahydro-4H-thiopyran End-Capped Oligo(cyclohexylidenes)
}

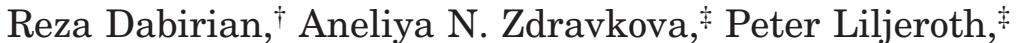 \\ Cornelis A. van Walree, ${ }^{\dagger}$ and Leonardus W. Jenneskens ${ }^{*, \dagger}$ \\ Department of Organic Chemistry and Catalysis, Debye Institute, Utrecht University, \\ Padualaan 8, 3584 CH Utrecht, The Netherlands, and Department of Condensed Matter and \\ Interfaces, Debye Institute, Utrecht University, Princetonplein 1, \\ 3508 TA Utrecht, The Netherlands
}

Received June 13, 2005. In Final Form: August 22, 2005

\begin{abstract}
Single-component and mixed self-assembled monolayers (SAMs) of one- and three-ring semirigid tetrahydro- $4 H$-thiopyran end-capped oligo(cyclohexylidenes)-that is, thiopyran (1), 4-(4-cyclohexylidenecyclohexylidene)tetrahydro- $4 H$-thiopyran (2), and 4-(tetrahydro- $4 H$-thiopyran-4-cyclohexylidene- 4 '-ylidene)tetrahydro- $4 H$-thiopyran (3) - on $\mathrm{Au}(111)$ substrates have been prepared and studied by cyclic voltammetry (CV), atomic force microscopy (AFM), and scanning tunneling microscopy (STM). It was found that the shortest adsorbate $\mathbf{1}$ more readily forms a SAM than $\mathbf{2}$ or $\mathbf{3}$. Notwithstanding, the SAMs of $\mathbf{2}$ or $\mathbf{3}$ are thermodynamically more stable due to favorable intermolecular attractions. Holes were made with the AFM tip establishing tilt angles of $30-50^{\circ}$ with respect to the surface normal for all SAMs. STM imaging showed well-ordered, line-shaped packing patterns with molecular resolution for the SAM of 2. Similar patterned structures were not observed for $\mathbf{1}$ and 3. Mixed SAMs were prepared by exposing a SAM of $\mathbf{1}$ to ethanol solutions of either $\mathbf{2}$ or 3. STM imaging revealed that domains of molecules of $\mathbf{2}$ or $\mathbf{3}$ amidst a monolayer of $\mathbf{1}$ are formed in both cases. Whereas in the mixed SAM of $\mathbf{1}$ and $\mathbf{2}$ the domains are irregularly shaped, circular islands of uniform size are found in the mixed SAM of $\mathbf{1}$ and $\mathbf{3}$.
\end{abstract}

\section{Introduction}

Molecules containing thiol and sulfide moieties readily adsorb onto metal substrates to form self-assembled monolayers (SAMs). These functionalities have been extensively employed for the anchoring of functionalized materials to, for example, $\mathrm{Au}(111)$ surfaces. ${ }^{1}$ In contrast to the vast amount of SAMs prepared from flexible adsorbates, there are relatively few SAMs prepared from (semi)rigid adsorbates. Examples include mercaptobiphenyls, ${ }^{2,3}$ (functionalized) [n]-staffane- $3,3^{(n-1)}$-dithiols, ${ }^{4}$ thioaromatics, ${ }^{5,6}$ polynorbornyldithiols,,${ }^{7,8} \alpha$-functionalized terthiophenes, ${ }^{9}$ and $\pi$-conjugated phenylene-ethylenes embedded in a dodecanethiol monolayer..$^{10}$ In the latter, the $\pi$-conjugated adsorbates function as electronically

* Corresponding author: tel +31 302533128; fax +31 302534533; e-mail l.w.jenneskens@chem.uu.nl.

Department of Organic Chemistry and Catalysis.

$¥$ Department of Condensed Matter and Interfaces.

(1) Love, J. C.; Estroff, L. A.; Kriebel, J. K.; Nuzzo, R. G.; Whitesides, G. M. Chem. Rev. 2005, 105, 1103-1169.

(2) Ulman, A.; Kang, J. F.; Shnidman, Y.; Liao, S.; Jordan, R.; Cjoi, G. Y.; Zaccaro, J.; Myerson, A. S.; Rafailovich, M.; Sokolov, J.; Fleischer, C. Rev. Mol. Biotechnol. 2000, 74, 175-188.

(3) Ulman, A. Acc. Chem. Res. 2001, 34, 855-863.

(4) Obeng, Y. S.; Laing, M. E.; Friedli, A. C.; Yang, H. C.; Wang, D.; Thulstrup, E. W.; Bard, A. J.; Michl, J. J. Am. Chem. Soc. 1992, 114, 9943-9952

(5) Sabatini, E.; Cohen-Boulakia, J.; Bruening, M.; Rubenstein, I. Langmuir 1993, 9, 2974-2981.

(6) Tao, Y. T.; Wu, C. C.; Eu, J. Y.; Lin, W. L.; Wu, K. C.; Chen, C. H. Langmuir 1997, 13, 4018-4023.

(7) Black, A. J.; Wooster, T. T.; Geiger, W. E.; Paddon-Row, M. N. J. Am. Chem. Soc. 1993, 115, 7924-7925.

(8) Wooster, T. T.; Geiger, W. E.; Oliver, A. M.; Black, A. J.; Craig, D. C.; Paddon-Row, M. N. Langmuir 1996, 12, 6616-6626.

(9) Liedberg, B.; Yang, Z.; Enquist, I.; Wirde, M.; Gelius, U.; Götz, G.; Bäuerle, P.; Rummel, R. M.; Ziegler, C.; Göpel, W. J. Phys. Chem. $B$ 1997, 101, 5951-5962.

(10) Bumm, L. A.; Arnold, J. J.; Cygan, M. T.; Dunbar, T. D.; Burgin, T. P.; Jones, L., II; Allara, D. L. Science 1996, 271, 1705-1707. active spacers that mediate tunneling processes and intramolecular charge transport phenomena.

It is generally accepted that flexible organic adsorbates tend to produce well-mixed SAMs ${ }^{11-17}$ in which phase segregation into macroscopic islands does not occur. Longchain alkanethiols are preferentially adsorbed compared with shorter derivatives due to the improved packing of the longer alkyl chains. ${ }^{17}$ (Semi)rigid adsorbates, however, do not mix together as well as the flexible ones, which may lead to domain formation. It is therefore attractive to employ rigid adsorbates for the preparation of mixed SAMs that consist of defined domains. In one of the few reported examples of mixed SAMs containing rigid adsorbates, various 4 '-substituted biphenyl thiols have been employed. ${ }^{18}$

Oligo(cyclohexylidenes) consist of cyclohexyl-type rings mutually connected at the 1 - and 4 - positions via olefinic bonds. ${ }^{19}$ They are available in length increments of ca. 0.4 $\mathrm{nm}$, by varying the number of cyclohexyl rings incorporated in each molecule. Single-crystal X-ray structures of (functionalized) oligo(cyclohexylidenes) have shown that they almost exclusively adopt an anti conformation in the

(11) Wang, Z.; Shi, Y. S.; Li, H. L. Can. J. Chem. 2001, 79, 328-336.

(12) Li, L.; Chen, S. Jiang, S. Langmuir 2003, 19, 666-671.

(13) Li, L.; Chen, S.; Jiang, S. Langmuir 2003, 19, 3266-3271.

(14) Shon, Y. S.; Lee, S.; Perry, S. C.; Lee, T. R. J. Am. Chem. Soc. 2000, 122, 1278-1281.

(15) Lestelius, M.; Engquist, I.; Tengvall, P.; Chaudhury, M. K.; Liedberg, B. Colloids Surf. B 1999, 15, 57-70.

(16) Shevade, A. V.; Zhou, J.; Zin, M. T.; Jiang, S. Langmuir 2001 17, 7566-7572.

(17) Laibinis, P. E.; Nuzzo, R. G.; Whitesides, G. M. J. Phys. Chem. 1992, 96, 5097-5105.

(18) Kang, J. F.; Liao, S.; Jordan, R.; Ulman, A. J. Am. Chem. Soc. 1998, 120, 9662-9667.

(19) Hoogesteger, F. J.; Havenith, R. W. A.; Zwikker, J. W.; Jenneskens, L. W.; Kooijman, H.; Veldman, N.; Spek, A. L. J. Org. Chem. 1995, $60,4375-4384$. 
Chart 1. Compounds 1-3

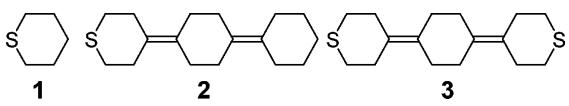

solid state. ${ }^{19,20}$ Hence, they possess a rodlike structure enabling columnar stacking in the solid state. Notwithstanding, the $\mathrm{sp}^{2}$ hybridized carbon atoms present in the cyclohexyl-type rings lower the barrier for ring inversion, ${ }^{19,21,22}$ rendering oligo(cyclohexylidenes) semiflexible in solution. This makes them good candidates for the preparation of mixed SAMs. An additional attractive aspect, especially of tetrahydro- $4 H$-thiopyran end-capped oligo(cyclohexylidenes), is that they exhibit an alternating $\sigma-\pi$ orbital topology, which enables them to relay electronic interactions via a through-bond orbital mechanism. ${ }^{23-25}$ Hence, various functionalized oligo(cyclohexylidenes) have already found application in molecular electronics and as active spacers in devices. ${ }^{25-28}$

Procedures for SAM formation of tetrahydro- $4 H$-thiopyran end-functionalized oligo(cyclohexylidenes) were previously reported..$^{25,29,30}$ It was shown that they form well-ordered SAMs, even when they consist of a single cyclohexane-type ring. Apparently, during SAM formation, the sulfide moiety (tetrahydro- $4 H$-thiopyran unit) of our end-functionalized oligo(cyclohexylidenes) interacts with the $\mathrm{Au}(111)$ surface through a dative bond by physisorption. ${ }^{1,31-33}$ The aim of the current study is 2 -fold. First, we want to provide a more detailed understanding of the mechanisms of SAM formation of tetrahydro- $4 H$-thiopyran end-capped oligo(cyclohexylidenes). Second, by using derivatives of different lengths, we aim to prepare mixed SAMs to study whether homogeneous mixing occurs or domains of the separate molecules are formed. This represents an initial step toward the preparation of patterned SAMs of tetrahydro- $4 H$-thiopyran end-capped oligo(cyclohexylidenes).

In this work, mixed layers of the one-ring thiopyran (1) with the three-ring 4-(4-cyclohexylidene-cyclohexylidene)tetrahydro- $4 H$-thiopyran $(2)$ and 4 -(tetrahydro- $4 H$-thiopyran-4-cyclohexylidene-4'-ylidene)tetrahydro- $4 H$-thiopyran (3) (Chart 1) are prepared by molecular exchange

(20) Veldman, N.; Spek, A. L.; Hoogesteger, F. J.; Zwikker, J. W.; Jenneskens, L. W. Acta Crystallogr. 1994, C50, 742-744.

(21) Grieg, J. T. J. Am. Chem. Soc. 1968, 90, 1065-1066. 067.

(23) Marsman, A.W.; Havenith, R. W. A.; Bethke, S.; Jenneskens, L. W.; Gleiter, R.; van Lenthe, J. H.; Lutz, M.; Spek, A. L. J. Org. Chem. 2000, 65, 4584-4592.

(24) Marsman, A.W.; Havenith, R. W. A.; Bethke, S.; Jenneskens, L. W.; Gleiter, R.; van Lenthe, J. H. Eur. J. Org. Chem. 2000, 14, 26292641 .

(25) Hoogesteger, F. J.; Van Walree, C. A.; Jenneskens, L. W.; Roest M. R.; Verhoeven, J. W.; Schuddeboom, W.; Piet, J. J.; Warman, J. M. Chem. Eur. J. 2000, 6, 2948-2959.

(26) Bakkers, E. P. A. M.; Marsman, A. W.; Jenneskens, L. W.; Vanmaekelbergh, D. Angew. Chem., Int. Ed. 2000, 39, 2297-2299.

(27) Oosterbaan, W. D.; Koper, C.; Braam, T. W.; Hoogesteger, F. J.; Piet, J. J.; Jansen, B. A. J.; van Walree, C. A.; van Ramesdonk, H. J.; Goes, M.; Verhoeven, J. W.; Schuddeboom, W.; Warman, J. M.; Jenneskens, L. W. J. Phys. Chem. A 2003, 107, 3612-3624.

(28) Oosterbaan, W. D.; van Gerven, P. C. M.; van Walree, C. A.; Koeberg, M.; Piet, J. J.; Havenith, R. W. A.; Zwikker, J. W.; Jenneskens,

L. W.; Gleiter, R. Eur. J. Org. Chem. 2003, 3117-3130.

(29) Bakkers, E. P. A. M.; Roest, A. L.; Marsman, A. W.; Jenneskens, L. W.; De Jong-van Steensel, L. I.; Kelly, J. J.; Vanmaekelbergh, D. J. Phys. Chem. B 2000, 104, 7266-7272.

(30) Reincke, F.; Hickey, S. G.; Kelly, J. J.; Braam, T. W.; Jenneskens, L. W.; Vanmaekelbergh, D. J. Electroanal. Chem. 2002, 522, 2-10.

(31) Lavrich, D. J.; Wetterer, S. M.; Bernasek, S. L.; Scoles, G. J.

Phys. Chem. B 1998, 102, 3456-3465.

(32) Takiguchi, H.; Sato, K.; Ishida, T.; Abe, K.; Yase, K.; Tamada, L. Langmuir 2000, 16, 1703-1710.

(33) Beulen, M. W. J.; Kastenberg, M. I.; van Veggel, F. C. J. M.; Reinhoudt, D. N. Langmuir 1998, 14, 7463-7467. of a SAM of $\mathbf{1}$ with either $\mathbf{2}$ or $\mathbf{3}$. The structure of the SAMs is assessed by a comparison of cyclic voltammograms (CV) and atomic force microscopy (AFM) images obtained for single-component SAMs with those obtained for the mixed SAMs. With the AFM tip, molecules in the assembly are mechanically removed in order to estimate the thickness of the monomolecular layers ${ }^{34}$ and the tilt angle between the molecular adsorbate and the $\mathrm{Au}(111)$ surface normal. Note that in the case of alkanethiols the thickness of the monolayer increases linearly with the number of methylene groups in the chain. ${ }^{35-37}$ Scanning tunneling microscopy (STM) is applied in order to achieve increased resolution and to access the molecular structure of the single-component layers and especially the mixed layers.

\section{Experimental Section}

General. Thin films of polycrystalline gold on borosilicate glass were flame-annealed in a hydrogen flame to promote the formation of $\mathrm{Au}(111)$ facets. This was followed by cooling under a flow of argon. The samples were then placed in sealed vials containing the incubation solution and stored in an oven at 60 ${ }^{\circ} \mathrm{C}$ for the required amount of time. Incubation solutions were prepared by dissolving a known amount of the adsorbate in absolute ethanol (Riedel-de Haën, $\geq 99.8 \%$ ). Compound 1 readily dissolved in ethanol, whereas samples of $\mathbf{2}$ and $\mathbf{3}$ had to be sonicated to obtain near-saturated solutions (ca. $0.3 \mathrm{mM}$ ). After incubation, the samples were successively rinsed with distilled water and ethanol prior to CV and AFM measurements. All CV, AFM, and STM samples were prepared and measured at least twice in order to ascertain the reproducibility of procedures and results. Thiopyran 1 (98\%, Lancaster) was carefully distilled under vacuum prior to use. Compounds $2^{19}$ and $3^{23}$ were prepared by previously reported methods.

Cyclic Voltammetry. Measurements were performed with an EG\&G Princeton Applied Research 366A potentiostat operated by a homemade Labview program. A three-electrode cell was used, equipped with a platinum counterelectrode $\left(8 \mathrm{~cm}^{2}\right)$, a saturated calomel electrode (SCE) as a reference, and an $\mathrm{Au}$ working electrode (effective geometrical area $0.24 \mathrm{~cm}^{2}$ ). The electrolyte solution consisted of $\mathrm{Fe}\left(\mathrm{CN}_{6}\right)^{3-/ 4-}(10 \mathrm{mM})$ as the electrochemical probe. A KCl (1 M) solution was used as the carrier electrolyte in high-purity water. Cyclic voltammograms were recorded at $25{ }^{\circ} \mathrm{C}$ between -0.2 and $0.6 \mathrm{~V}$ vs SCE with a constant scan rate of $100 \mathrm{mV} / \mathrm{s}$, starting at $0 \mathrm{~V}$ with a potential increase. Reproducible cyclic voltammograms were obtained during repeated consecutive scans for each SAM; the third scan was recorded. Note that it is documented that SAMs derived from alkyl sulfides on $\mathrm{Au}(111)$ are electrochemically stable between -0.4 and $0.8 \mathrm{~V}$ vs SCE. ${ }^{33}$

AFM Measurements. SAMs were examined by AFM within $3 \mathrm{~h}$ after removal of the substrate from the incubation solution. Imaging was done with a Nanoscope III (Digital Instruments) in contact mode with an oxide-sharpened silicon nitride tip $(k=$ $0.12 \mathrm{~N} / \mathrm{m}$ ) at ambient temperature. The AFM was equipped with an $\mathrm{E}$ scanner. The maximum area that can be observed is $12 \times$ $12 \mu \mathrm{m}^{2}$. The $Z$ limit is $3.542 \mu \mathrm{m}$. The thickness of the monolayers was measured by removing a part of the monolayer with the AFM tip. To this end the tip was moved downward and pressed on top of the adsorbed molecules with large force $(1-20 \mathrm{nN})$.

The roughness parameters given are average values of the root-mean-square (rms) of the height of several scans from at least two separate images of each monolayer type. The area of each scan was $120 \mathrm{~nm}^{2}$. The sections scanned were chosen at positions in the center of the Au plateaus, as far as possible from the edges, to minimize the effect of irregularities of the underlying Au surface.

STM Measurements. STM images were obtained with a Nanoscope III (Digital Instruments). Both electrochemically

(34) Han, T.; Beebe, T. P., Jr. Langmuir 1994, 10, 2705-2709.

(35) Porter, M. D.; Bright, T. B.; Allara, D. L.; Chidsey, C. E. D. J. Am. Chem. Soc. 1987, 109, 3559-3568.

(36) Bain, C. D.; Troughton, E. B.; Tao, Y. T.; Evall, J.; Whitesides, G. M.; Nuzzo, R. G. J. Am. Chem. Soc. 1989, 111, 321-335.

(37) Nuzzo, R. G.; Dubois, L. H.; Allara, D. L. J. Am. Chem. Soc. 1990, $112,558-569$ 


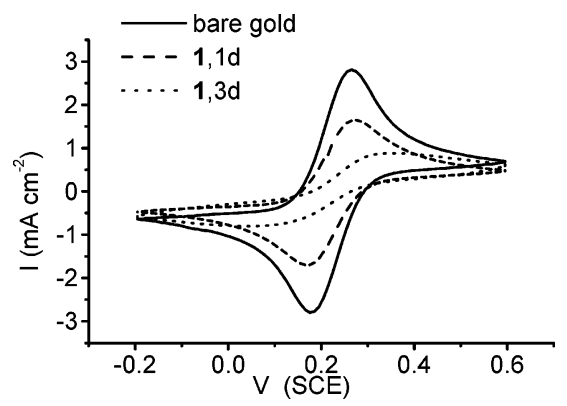

Figure 1. CVs of bare gold and of 1 after 1 and 3 days of incubation.

etched and cut $\mathrm{Pt} / \mathrm{Ir}$ tips were used. The images were recorded with set-point currents of $25-30 \mathrm{pA}$ and a bias of $0.25 \mathrm{~V}$. The images were processed with WSxM free software, downloadable at http://www.nanotec.es.

\section{Results and Discussion}

Single-Component SAMs. The quality of the SAMs of $\mathbf{1}-\mathbf{3}$ was assessed by their ability to block heterogeneous electron transfer between the $\mathrm{Au}(111)$ surface and the $\mathrm{Fe}\left(\mathrm{CN}_{6}\right)^{3-/ 4-}$ redox probe, measured by cyclic voltammetry. The shape of the cyclic voltammograms and the measured current densities yield information about the formation and organization of the monolayers. As a reference, the bare gold electrode shows quasireversible behavior with a peak separation of ca. $90 \mathrm{mV}$. This is in agreement with fast redox kinetics with respect to the applied scan rate $(100 \mathrm{mV} / \mathrm{s})$.

In the case of $\mathbf{1}$ an $\mathrm{Au}$ electrode, which had been immersed in a $15 \mathrm{mM}$ ethanol solution of $\mathbf{1}$ for 1 day at $60{ }^{\circ} \mathrm{C}$, showed a current reduction of ca. $50 \%$ and a peak separation of $110 \mathrm{mV}$ (Figure 1, Table 1). Incubation for 3 days gave a somewhat larger current reduction $(60 \%)$ and a peak separation of $300 \mathrm{mV}$. Thus, the electron transfer reaction of the redox probe becomes slower due to SAM formation. It should be noted that the degree of current reduction did not markedly change with an increase in the concentration of $\mathbf{1}$ in the immersion solution. CVs obtained for $\mathrm{Au}(111)$ substrates with either $15 \mathrm{mM}$ or $90 \mathrm{mM}$ ethanol solutions of 1 gave similar results. Apparently, the $15 \mathrm{mM}$ concentration is already above the limiting concentration of the adsorbate, and SAM formation at this stage is independent of the incubation concentration; that is, the kinetics of the adsorption step control the rate of monolayer formation.

Whereas $\mathbf{1}$ is readily soluble in ethanol, the solubility of $\mathbf{2}$ and $\mathbf{3}$ in this solvent is much lower. Therefore, nearsaturated solutions of $\mathbf{2}$ and $\mathbf{3}$ (ca. $0.3 \mathrm{mM}$ ) were used to incubate the substrate at $60{ }^{\circ} \mathrm{C}$. A current reduction of $30 \%$ and peak separation of $100 \mathrm{mV}$ is obtained for 2 after 6 days, which increases to $50 \%$ after 9 days with a peak separation of $170 \mathrm{mV}$ (Figure 2, Table 1). For compound 3, a $35 \%$ reduction of the current and a $110 \mathrm{mV}$ peak separation is obtained after incubation for 3 days. After 6 days the current reduction increases to $45 \%$ with a peak separation of $130 \mathrm{mV}$ (cf. Supporting Information, Figure S6).

The lower degree of current reduction and peak separation observed with the three-ring derivatives $\mathbf{2}$ and $\mathbf{3}$ compared with that of $\mathbf{1}$ suggest that surface coverage is less dense in the case of $\mathbf{2}$ and 3 . Given the similar solubility of $\mathbf{2}$ and $\mathbf{3}$ in ethanol, the more facile SAM formation of $\mathbf{3}$ compared to that of $\mathbf{2}$ is rationalized by the fact that $\mathbf{3}$ contains two tetrahydro- $4 H$-thiopyran end groups, whereas 2 contains only one (Chart 1 ). Hence with more possibilities to interact with the $\mathrm{Au}(111)$ surface, SAM formation is expected to be faster for 3 .

Next, the single-component SAMs were studied by contact-mode AFM; images were taken at various scan sizes. The degree of roughness is applied as a measure for the extent of surface coverage, viz., to follow the monolayer formation process. Qualitatively, roughness seen at low magnification ( $5 \mu \mathrm{m}^{2}$ area scanned) indicates more dense coverage than roughness seen at high magnification $(<1$ $\mu \mathrm{m}^{2}$ area scanned). The roughness can be assessed semiquantitatively by the roughness parameter (rms), which is determined from the average height differences of several scans with identical areas from at least two separate images of each monolayer type. ${ }^{38,39}$ As will be shown below, the rms parameter provides a description of general trends such as the monolayer formation process itself, as well as the occurrence of exchange processes during the preparation of mixed SAMs. Finally, in order to measure the layer thickness, holes were made in the samples by removing a part of the monolayer with the AFM tip. ${ }^{34}$

AFM images of SAMs of 1 were recorded after incubation for 1,2 , and 3 days at various scan sizes (cf. Supporting Information, Figure S1). The images taken after 1 and 2 days show a relatively smooth surface at low magnifications. In the 1-day sample, the presence of a layer was observed only with higher magnification. Pinholes were also found, indicating that at this stage the coverage is still not optimal. The roughness parameter was determined to be $0.08 \pm 0.01 \mathrm{~nm}$ (Table 1 ). The 3 -day sample showed coverage even at low magnification, and the roughness parameter increased to $0.54 \pm 0.05 \mathrm{~nm}$. The increase of the roughness parameter confirms SAM formation in the case of $\mathbf{1}$. Note, however, that the roughness parameter for $\mathbf{1}$ is expected to be too high since molecules of $\mathbf{1}$ are readily removed from the surface by the AFM tip (vide infra). Holes made by repetitive scanning revealed a depth of $0.3-0.4 \mathrm{~nm}$ (Table 1 , Figure 3 ). This value is in agreement with the length of $\mathbf{1}$, which was estimated to be $0.32 \mathrm{~nm}$ (ab initio RHF/6-31G* calculation). ${ }^{24}$ STM images of $\mathbf{1}$ after 3 days show complete substrate coverage; however, molecular resolution could not be achieved (Figure 4).

AFM images of the SAM of $\mathbf{2}$ indicate a low degree of coverage even after incubation for 6 days, with a roughness parameter of $0.07 \pm 0.01 \mathrm{~nm}$. Coverage improved after a 9-day incubation period; the roughness parameter increases to $0.16 \pm 0.03 \mathrm{~nm}$ (Table 1 ). Examination of a hole made in the 9-day sample gave a layer thickness of $0.7-$ $0.8 \mathrm{~nm}$. Since the length of 2 is $1.15 \mathrm{~nm}$ (ab initio RHF/ 6-31G* calculations), ${ }^{23}$ the difference between the hole depth and molecular length suggests that the adsorbate is tilted with respect to the surface normal (tilt angle 30$50^{\circ}$ ). STM images show well-ordered line-shaped patterned structures in which individual molecules appear to be positioned in parallel rows (Figure 5). A cross-section line with a length of $0.23 \mathrm{~nm}$ over six adjacent molecules indicated a distance of ca. $0.5 \mathrm{~nm}$ between each molecule (Figure 5). This value is in good agreement with the width of the unit cell in the single-crystal X-ray structure of 4,4'-bis(tetrahydro- $4 H$-thiopyran-4-cyclohexylidene- $4^{\prime}$ ylidene), that is, a lower homologue of $3 .^{23}$

(38) Hegner, M.; Wagner, P.; Semenza, G. C. Surf. Sci. 1993, 291 $39-46$.

(39) Although hitherto AFM rms parameters have been primarily used for the characterization of changing inorganic surfaces, they can also be applied to assess exchange processes in our organic SAMs. However, since rms values for the SAMs are ca. 1 order of magnitude smaller than in conventional applications, they can only be used to distinguish trends. 
Table 1. Salient Data for SAMs of Compounds 1-3 Obtained by CV and AFM

\begin{tabular}{|c|c|c|c|c|c|c|}
\hline compd & $\begin{array}{c}\text { incubation } \\
\text { time } \\
\text { (days) }\end{array}$ & $\begin{array}{c}\text { incubation } \\
\text { concn } \\
(\mathrm{mM})\end{array}$ & $\begin{array}{l}\text { current } \\
\text { reduction } \\
(\%)\end{array}$ & $\begin{array}{c}\text { peak } \\
\text { separation } \\
(\mathrm{mV})\end{array}$ & $\begin{array}{l}\text { hole } \\
\text { depth } \\
(\mathrm{nm})\end{array}$ & $\begin{array}{l}\text { roughness } \\
\text { parameter }^{a} \\
(\mathrm{rms})(\mathrm{nm})\end{array}$ \\
\hline 1 & 1 & 15 & 50 & 110 & $0.28-0.38$ & $0.08 \pm 0.01$ \\
\hline 1 & 3 & 15 & 60 & 300 & $0.28-0.38$ & $0.54 \pm 0.05$ \\
\hline 2 & 6 & 0.3 & 30 & 100 & & $0.07 \pm 0.01$ \\
\hline 2 & 9 & 0.3 & 50 & 170 & $0.65-0.90$ & $0.16 \pm 0.03$ \\
\hline $\mathbf{3}$ & 3 & 0.3 & 35 & 110 & $0.75-0.90$ & $0.13 \pm 0.04$ \\
\hline 3 & 6 & 0.3 & 45 & 130 & & $0.15 \pm 0.03$ \\
\hline $\mathbf{1}+\mathbf{2}$ & $3+6$ & $15+0.3$ & 40 & 100 & $0.60-0.70$ & $0.29 \pm 0.09$ \\
\hline $1+2$ & $3+9$ & $15+0.3$ & 40 & 150 & $0.60-0.70$ & $0.13 \pm 0.03$ \\
\hline $\mathbf{1}+\mathbf{3}$ & $3+3$ & $15+0.3$ & 25 & 100 & $0.60-0.80$ & $0.14 \pm 0.06$ \\
\hline $\mathbf{1}+\mathbf{3}$ & $3+6$ & $15+0.3$ & 35 & 100 & $0.60-0.80$ & $0.11 \pm 0.02$ \\
\hline
\end{tabular}

${ }^{a}$ All scan sizes were taken at $120 \mathrm{~nm}^{2}$.

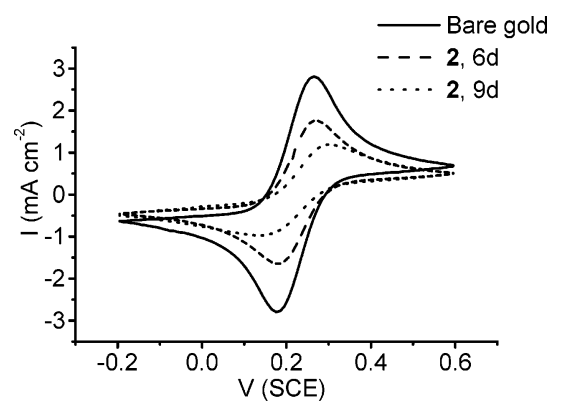

Figure 2. CVs of bare gold and of 2 after 6 and 9 days of incubation.
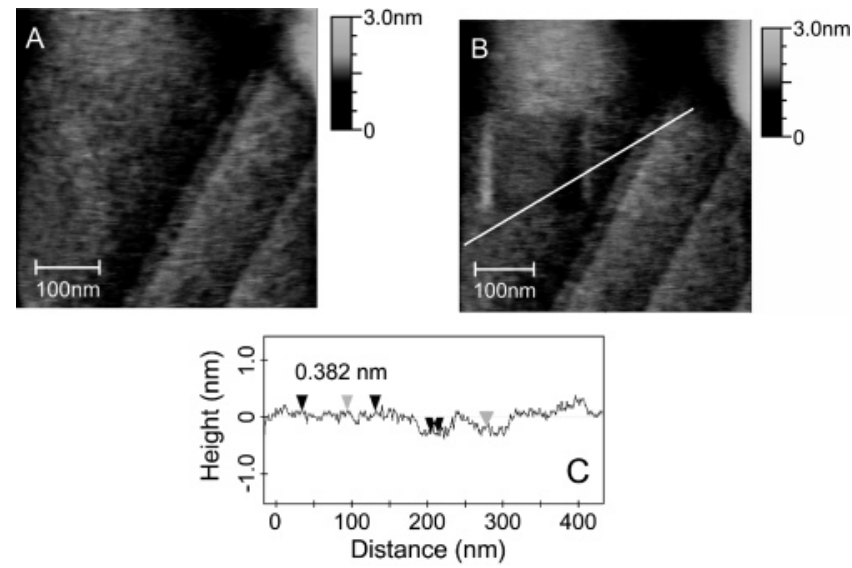

Figure 3. AFM height images of $\mathbf{1}$ after 1 day of incubation, before (A) and after (B) hole making, and a cross-section profile (C).

Compound 3 (length $1.16 \mathrm{~nm}$, ab initio RHF/6-31G* calculations) ${ }^{23}$ exhibits similar assembly behavior; increased coverage is observed with longer incubation times. However, the improvement in the quality of the SAM is less pronounced. This is exemplified by the STM images of the SAM of 3, which lacks the well-ordered patterns found for the SAM of 2 (Supporting Information, Figure S4; cf. also Figure 5). Holes made with the AFM tip in the 3 -day sample of $\mathbf{3}$ gave a layer thickness ranging from 0.8 to $0.9 \mathrm{~nm}$ (cf. Supporting Information, Figure S3), suggesting that the adsorbate $(\mathbf{3})$ is tilted with an angle of $30-50^{\circ}$ with respect to the surface normal. No significant difference of the roughness parameter, which increases from $0.13 \pm 0.04 \mathrm{~nm}$ after 3 days to $0.15 \pm 0.03 \mathrm{~nm}$ after 6 days, was found (Table 1 ).

Mechanical removal of the adsorbates from the surface with the AFM tip proved to be considerably more straightforward for the SAM of 1 than for the SAMs of either $\mathbf{2}$ or $\mathbf{3}$. Whereas $\mathbf{1}$ could be removed with just a few repetitive scans and a force of $1 \mathrm{nN}$, the longer compounds $\mathbf{2}$ and $\mathbf{3}$ after deposition required an applied force of up

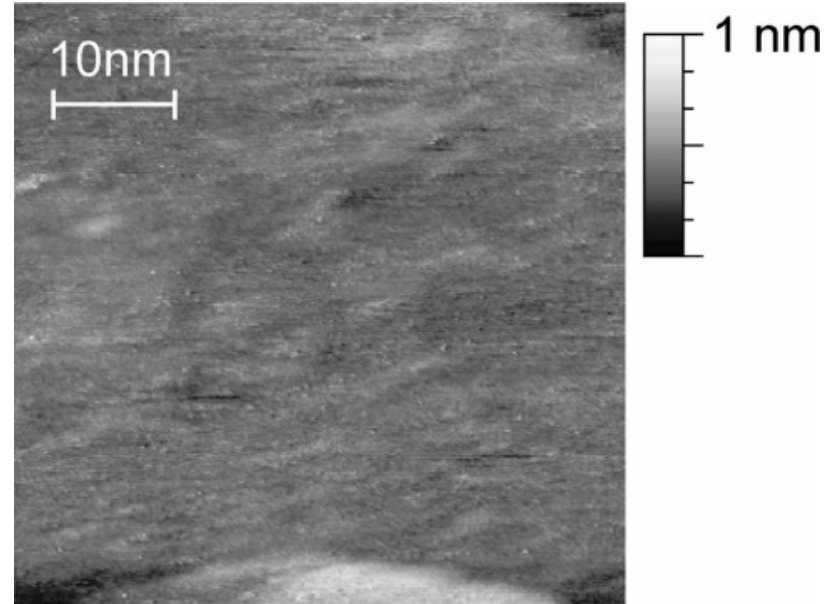

Figure 4. STM image of $\mathbf{1}$ after 3 days.

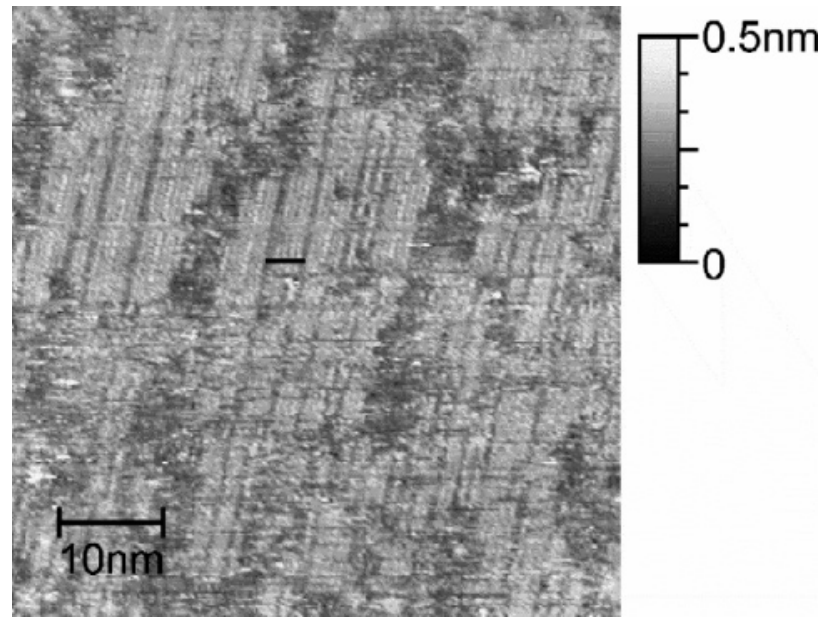

Figure 5. STM height image of 2 after 9 days of incubation, showing well-ordered packing patterns (note the presence of the cross-section line; see text).

to $20 \mathrm{nN} .{ }^{40}$ This suggests that 2 or 3 interact more strongly after physisorption to the $\mathrm{Au}(111)$ surface. This can be rationalized by considering the single-crystal X-ray structure of end-functionalized oligo(cyclohexylidenes), ${ }^{19,20}$ from which it is clear that they stack on top of each other to form well-ordered structures in the solid state. The intermolecular forces that govern the packing structure become more pronounced with each added cyclohexyl-like ring. Consequently, increasing force is needed to remove the longer adsorbates from the surface.

(40) De Jong-van Steensel, L. I.; Snel, M. M. E.; van den Berg, G. J. K.; Jenneskens, L. W.; van der Eerden, J. P. J. M. Cryst. Growth Des. 2001, 5, 347-353. 
In short, the longer derivatives $\mathbf{2}$ and $\mathbf{3}$ need more time to form a SAM and are kinetically less preferred. Thus, after annealing, the structure induced by the intermolecular forces renders SAMs of $\mathbf{2}$ or $\mathbf{3}$ thermodynamically more stable than that of $\mathbf{1}$.

Mixed-Layer SAMs. The difference in solubility in the immersion solution between the short compound $\mathbf{1}$ and the extended homologues $\mathbf{2}$ and $\mathbf{3}$, together with the relative ease of SAM formation of $\mathbf{1}$ (3 days), indicates that exposure of a SAM of $\mathbf{1}$ to a solution of $\mathbf{2}$ or $\mathbf{3}$ may give access to mixed SAMs.

$\mathrm{CVs}$ of mixed-layer samples of $\mathbf{1}$ and $\mathbf{2}$ recorded after $3+6$ and $3+9$ days of incubation both showed a reduction in current of ca. 40\%, compared with the bare gold surface, and a peak separation of $100-150 \mathrm{mV}$ (Table 1). Samples were taken after the initial 3-day immersion in $\mathbf{1}$ to establish that a SAM of $\mathbf{1}$ had indeed formed before immersion into the solution of $\mathbf{2}$. The CVs of mixed $\mathbf{1}$ and 2 samples resemble single-component CVs of 2 after 6 days. The final extent of current reduction is less than that obtained after 3 days of immersion in $\mathbf{1}$ (current reduction of $60 \%$ and a peak separation of $300 \mathrm{mV}$ ). This indicates that molecular exchange has indeed taken place. However, even after 9 days of incubation of the mixed sample in a solution of $\mathbf{2}$, the current reduction did not reach the level that was found with a 9-day sample of 2 alone (cf. Supporting Information, Figure S7). Apparently, partial rather than complete exchange occurs.

CVs of mixed-layer samples of $\mathbf{1}$ and $\mathbf{3}$ recorded after $3+3$ and $3+6$ days of incubation showed a reduction in current of ca. $25 \%$ and $35 \%$, respectively, compared with the bare gold surface and a peak separation of $100 \mathrm{mV}$ (cf. Supporting Information, Figure S8, and Table 1). The current reduction and peak separation values are smaller than those found earlier for any of the single-component SAMs after an equal incubation time. Again, this suggests that a partial exchange process has taken place. Consistently, in both types of mixtures ( $\mathbf{1}$ and $\mathbf{2}$ as well as $\mathbf{1}$ and 3), an exchange process is observed and the mixed layers exhibit properties that are closer to those of the longer derivatives (Table 1). The lower final current reduction reflect a lower total density of coverage than in any of the single-component SAMs, which is attributed to less efficient packing due to the presence of different types of molecules. Since the CV results do not give any insight as to whether the mixed SAMs consist of either random mixtures or domains of the separate compounds, the samples were further characterized by AFM and STM.

Upon equal incubation time, AFM at low magnification $\left(>2 \mu \mathrm{m}^{2}\right.$ ) showed that the mixed layer of $\mathbf{1}$ and $\mathbf{2}$ as well as that of $\mathbf{1}$ and $\mathbf{3}$ appears to be smoother than the layers of single components $\mathbf{2}$ and $\mathbf{3}$, respectively (cf. Supporting Information, Figure S2). AFM images of mixed layers of 1 and 2 gave a roughness parameter of $0.29 \pm 0.09 \mathrm{~nm}$ after $3+6$ days of incubation (Table 1 ), which decreases to $0.13 \pm 0.03 \mathrm{~nm}$ after $3+9$ days. The mixed layer of 1 and 3 showed a roughness parameter of $0.14 \pm 0.06 \mathrm{~nm}$ after $3+3$ days, which decreases to $0.11 \pm 0.02 \mathrm{~nm}$ after $3+6$ days of incubation. In each case the final roughness value is slightly lower than that found for the SAMs of the respective three-ring components. These observations substantiate the argument that the distribution of adsorbates in the mixed layer is less dense. Moreover, the roughness parameter of the single-component SAMs increases in time, reflecting the buildup of a monolayer from an initially smooth gold surface. In contrast, the roughness parameters of the mixtures tend to decrease in time, reflecting the transformation from a SAM of pure $\mathbf{1}$ to that of one with primarily $\mathbf{2}$ or $\mathbf{3}$ character.
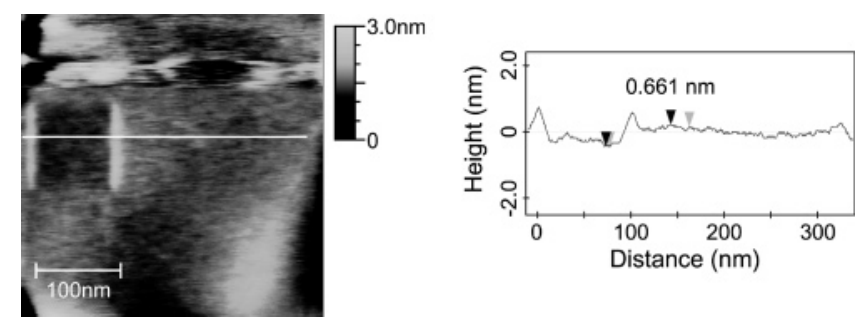

Figure 6. AFM height image of a mixture of $\mathbf{1}$ and $\mathbf{3}$ after 3 +6 days, after hole making. The thickness of the monolayer is given by the vertical distance between the markers on the corresponding cross-section profile.

Holes made with the AFM tip in the mixed layers by AFM were less deep than those made in the layers of the corresponding longer adsorbate. Values between 0.6 and $0.7 \mathrm{~nm}$ for the mixed layer of $\mathbf{1}$ and $\mathbf{2}$ (cf. Supporting Information, Figure S5) and $0.6-0.8 \mathrm{~nm}$ for the mixed layer of $\mathbf{1}$ and $\mathbf{3}$ were obtained (Figure 6). In addition, holes with a depth of only $0.3-0.4 \mathrm{~nm}$ were also found. The latter are presumably derived from detachment of $\mathbf{1}$ during reincubation. These results provide evidence that exchange has occurred. The values did not change with longer incubation times; holes made after $3+6$ days and $3+9$ days of incubation of the mixed $\mathbf{1}$ and $\mathbf{2}$ sample were found to be of equal depth. A similar result was obtained after $3+3$ and $3+6$ days of incubation of the mixed 1 and $\mathbf{3}$ sample. Although the measure of the force required to remove the adsorbates from the mixed-layer surface was not optimized in the case of the mixed layers, it was found to be close to the force needed to remove adsorbates from the single-component SAMs of $\mathbf{2}$ or $\mathbf{3}(20 \mathrm{nN})$. This further corroborates that molecular exchange has occurred.

In short, the roughness parameters, hole depths, and removal force values are all in agreement and suggest that the mixed layers exhibit characteristics that are more reminiscent of those determined for the longer derivatives.

The holes made with the AFM tip have a minimum size of $100 \times 100 \mathrm{~nm}^{2}$ and lack the resolution that is required to detect smaller domains of separate compounds. Therefore, the samples were subjected to STM. STM images of the mixed layers revealed small areas of inhomogeneities that were not detected by AFM. Samples of $\mathbf{1}$ and $\mathbf{2}$ that were incubated for $3+9$ days, although showing evidence of molecular exchange, did not include clearly identifiable domains (Figure 7A). The exchange process at that stage is incomplete. At a longer incubation time in the solution of $\mathbf{2}$, domain formation in the mixed layers of $\mathbf{1}$ and $\mathbf{2}$ was also observed by STM. In samples taken after $3+14$ days, irregular shaped domains of $\mathbf{2}$ separated by patches of $\mathbf{1}$ are clearly visible (Figure 7B). The sizes of the domains vary from 0.5 to $2.0 \mathrm{~nm}$. Cross-section profiles of this mixed sample show depressions of about $0.2-0.3 \mathrm{~nm}$. In contrast, images taken of the mixed layers of $\mathbf{1}$ and $\mathbf{3}$ after $3+3$ days showed depressions with a depth of $0.3-1.0 \mathrm{~nm}$ (Figure 8A), whereas the difference in length between 1 and 3 is ca. $0.8 \mathrm{~nm}$. This is evidence that the SAM is in the process of molecular exchange. However, the exchange process is still incomplete and the images are comparable to those of the $3+3$ day samples of $\mathbf{1}$ and $\mathbf{2}$ (Figure 7A). STM images taken after $3+6$ days show depressions of about $0.5-0.6 \mathrm{~nm}$ (Figure 8B). This narrowing down of depression depth suggests that the molecular exchange has taken its course and an optimal mixed layer has been reached. Hence the CV and AFM as well as STM results show that both long ( 2 or $\mathbf{3})$ and short (1) adsorbates are present in the case of the mixed SAMs. Moreover, it can be concluded that the structures visible in Figure $8 \mathrm{~B}$ 

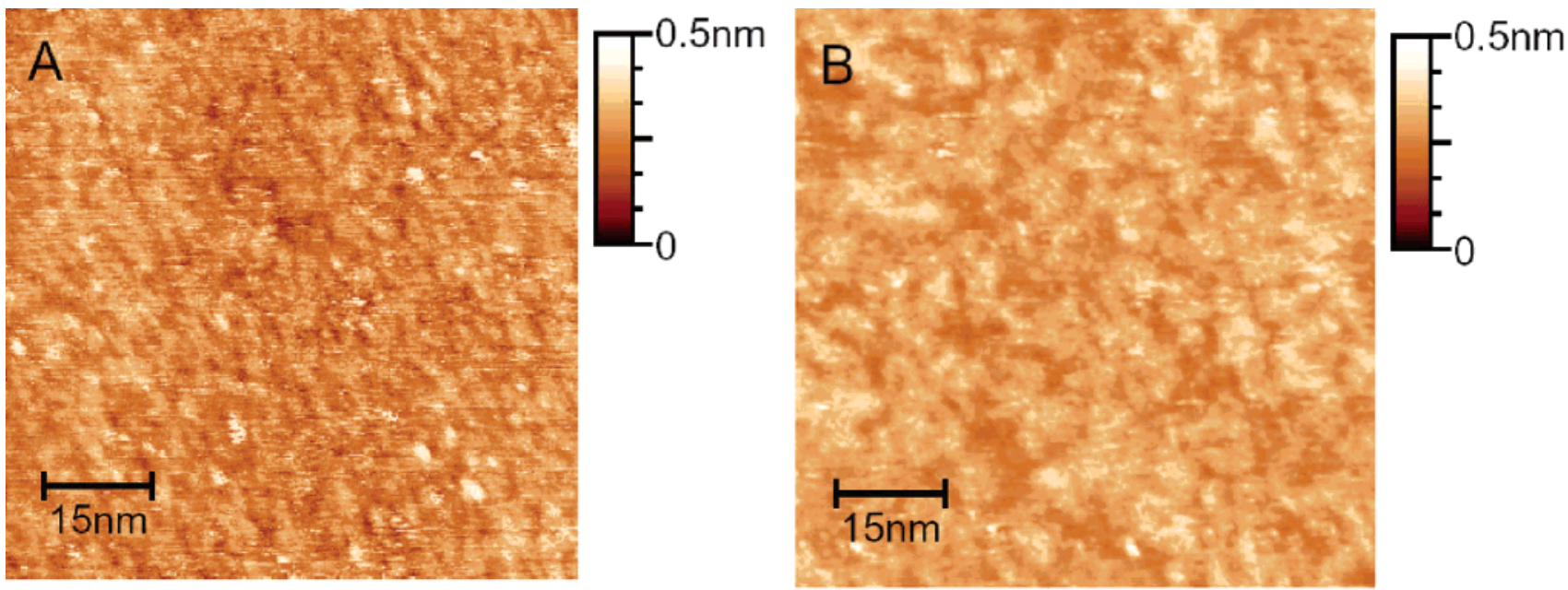

Figure 7. STM image of a mixed layer of 1 and $\mathbf{2}$ after $3+9$ days (A) and after $3+14$ days (B) (see also Figures 4 and 5 for single-component STM images).
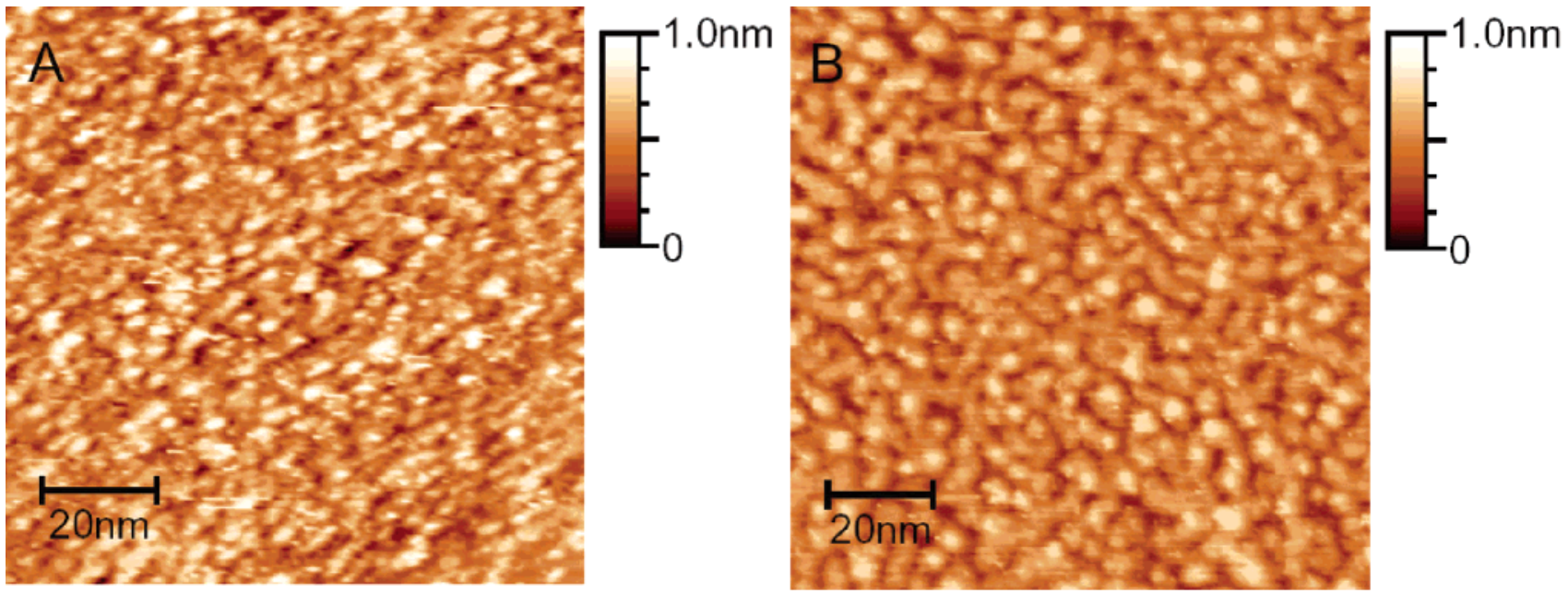

Figure 8. STM image of a mixed layer of 1 and $\mathbf{3}$ after $3+3$ days (A) and after $3+6$ days (B).

represent circular islands of $\mathbf{3}$ amidst a monolayer of $\mathbf{1}$. The width of the islands is nearly constant (diameter 5 $\mathrm{nm}$ ). If a distance of ca. $0.5 \mathrm{~nm}$ between molecules is assumed, as derived from the solid-state structure of tetrahydro- $4 H$-thiopyran end-functionalized oligo(cyclohexylidenes), ${ }^{19,20,23}$ this suggests that each island incorporates about 50-100 molecules of 3 .

The exchange process is apparently slower for the mixed SAM of $\mathbf{1}$ and $\mathbf{2}$ than for that of $\mathbf{1}$ and $\mathbf{3}$. Apparently, the lack of a second sulfide moiety in the case of $\mathbf{2}$ prolongs the rate of domain formation in the mixed SAM to a greater extent than it prolongs single-component SAM formation of $\mathbf{2}$. The additional time required to form defined mixed structures can be attributed to the various steps required in SAM formation. Comparative studies on alkanethiols ${ }^{41}$ suggested that an initial rapid stage in which the molecules are adsorbed on the surface is followed by a slower rearrangement step. This rearrangement cannot occur until the absorbates are attached to the surface. This process resembles Ostwald-type ripening, that is, the growth of larger crystals at the expense of smaller ones. ${ }^{42}$

\section{Conclusions}

We have carried out a study of SAMs of oligo(cyclohexylidenes) $\mathbf{1}-\mathbf{3}$ on $\mathrm{Au}(111)$ substrates by exploring the

(41) Yamada, R.; Uosaki, K. Langmuir 1998, 14, 855-861.

(42) ten Grotenhuis, E.; Marsman, A. W.; Hoogesteger, F. J.; van Miltenburg, J. C.; van der Eerden, J. P. J. M.; Jenneskens, L. W.; Smeets W. J. J.; Spek, A. L. J. Cryst. Growth 1998, 191, 834-845. micro- and macrostructure of the layer by CV, AFM, and STM. With AFM it was possible to monitor how the formation of the SAM evolves from the early stages, where pinholes are present, until optimal coverage is achieved for $\mathbf{1 - 3}$. The results show that SAM formation of $\mathbf{1}$ is kinetically preferred. Although SAM formation in the case of $\mathbf{2}$ and $\mathbf{3}$ is slower, their optimal monolayers after annealing are thermodynamically more stable. This is attributed to improved intermolecular interactions between their hydrocarbon skeletons. In line with this contention, hole making with the AFM tip in SAMs of 2 or 3 requires a 20-fold larger force than that needed for the SAM of 1. A layer thickness of $0.3-0.4 \mathrm{~nm}$ was found for the SAM of 1 and $0.6-0.9 \mathrm{~nm}$ for the SAMs of 2 and 3 , implying tilt angles of $30-50^{\circ}$ with respect to the surface normal for 1-3. STM imaging of the SAM of 2 revealed well-ordered, line-shaped packing patterns with molecular resolution. An intermolecular distance of ca. $0.5 \mathrm{~nm}$ is discernible, which corresponds to the width of the unit cell found in single-crystal X-ray structures of oligo(cyclohexylidenes). ${ }^{23}$ In contrast, patterned structures were not observed by STM for the SAMs of either 1 or 3.

Subsequently, mixed SAMs were prepared by incubating a SAM of $\mathbf{1}$ in solutions of $\mathbf{2}$ or $\mathbf{3}$. A comparison of the $\mathrm{CV}$ and AFM results of these mixed SAMs with those of the related single-component SAMs provided evidence of partial molecular exchange of $\mathbf{1}$ with either $\mathbf{2}$ or $\mathbf{3}$. STM images of the mixed SAMs of $\mathbf{1}$ and $\mathbf{2}$ as well as and $\mathbf{1}$ and 
3 show that, in these mixed systems, domain formation occurs. A survey of cross-section profiles of both mixed SAMs suggests that in both cases domains consisting of the longer derivative ( 2 or $\mathbf{3}$ ) are embedded amidst a monolayer of $\mathbf{1}$. Notwithstanding, the domains vary in shape and size. In the case of the mixed SAM of $\mathbf{1}$ and $\mathbf{2}$, the domains are irregularly shaped with varying lengths and widths. In the case of the mixed SAM of $\mathbf{1}$ and $\mathbf{3}$, however, the domains are circular islands of $\mathbf{3}$ of relatively uniform size (diameter of $5 \mathrm{~nm}$ ). Hence, each domain contains about 50-100 molecules of 3. Furthermore, the time required for molecular exchange to achieve domains in the mixed SAM of $\mathbf{1}$ and $\mathbf{2}$ is longer (14 days) than that required for SAM formation of 2 itself( 9 days). In contrast, molecular exchange and domain formation in the case of the mixed SAM of $\mathbf{1}$ and $\mathbf{3}$ is already completed after 6 days, which resembles SAM formation of $\mathbf{3}$ itself.

Acknowledgment. Karin Overgaag is acknowledged for her assistance with STM experiments. P.L. acknowledges financial support from the European Union (EU network "Nanospectra", HPRH-CT-2001-00320).

Supporting Information Available: AFM images of SAMs of 1, 2, and 3 (after hole making); STM image of a SAM of 3; AFM image of mixed SAM of 1 and 2; and CVs of SAMs of $\mathbf{3}$ and mixed layers of $\mathbf{1}$ and $\mathbf{2}$ as well as $\mathbf{1}$ and $\mathbf{3}$. This material is available free of charge via the Internet at http://pubs.acs.org. LA051570L 